\title{
Correction to: The rational design of regional regimes: contrasting Amazonian, Central African and Pan-European Forest Governance
}

\author{
Joana Carlos Bezerra ${ }^{1,2}$ (D) Jan Sindt ${ }^{3} \cdot$ Lukas Giessen $^{1,4}$
}

Published online: 12 September 2018

(c) Springer Nature B.V. 2018

\section{Correction to: Int Environ Agreements https://doi.org/10.1007/s10784-018-9395-z}

Mistakenly, a prior, premature version of this article was published online, which did not reflect revisions made based on a last round of external peer review. The original article has been corrected.

The original article can be found online at https://doi.org/10.1007/s10784-018-9395-z.

Joana Carlos Bezerra

bezerra.joana@gmail.com

Jan Sindt

jan.sindt@climateanalytics.org

Lukas Giessen

Lukas.Giessen@efi.int

1 Chair of Forest and Nature Conservation Policy, University of Göttingen, Büsgenweg 3, 37077 Göttingen, Germany

2 Department of Environmental Science, Rhodes University, Room 106, Bangor House, Sommerset Street, Grahamstown 6140, South Africa

3 Climate Analytics, Friedrichstraße 231, Haus B, 10969 Berlin, Germany

4 European Forest Institute (EFI), Bonn Office, Bonn, Germany 